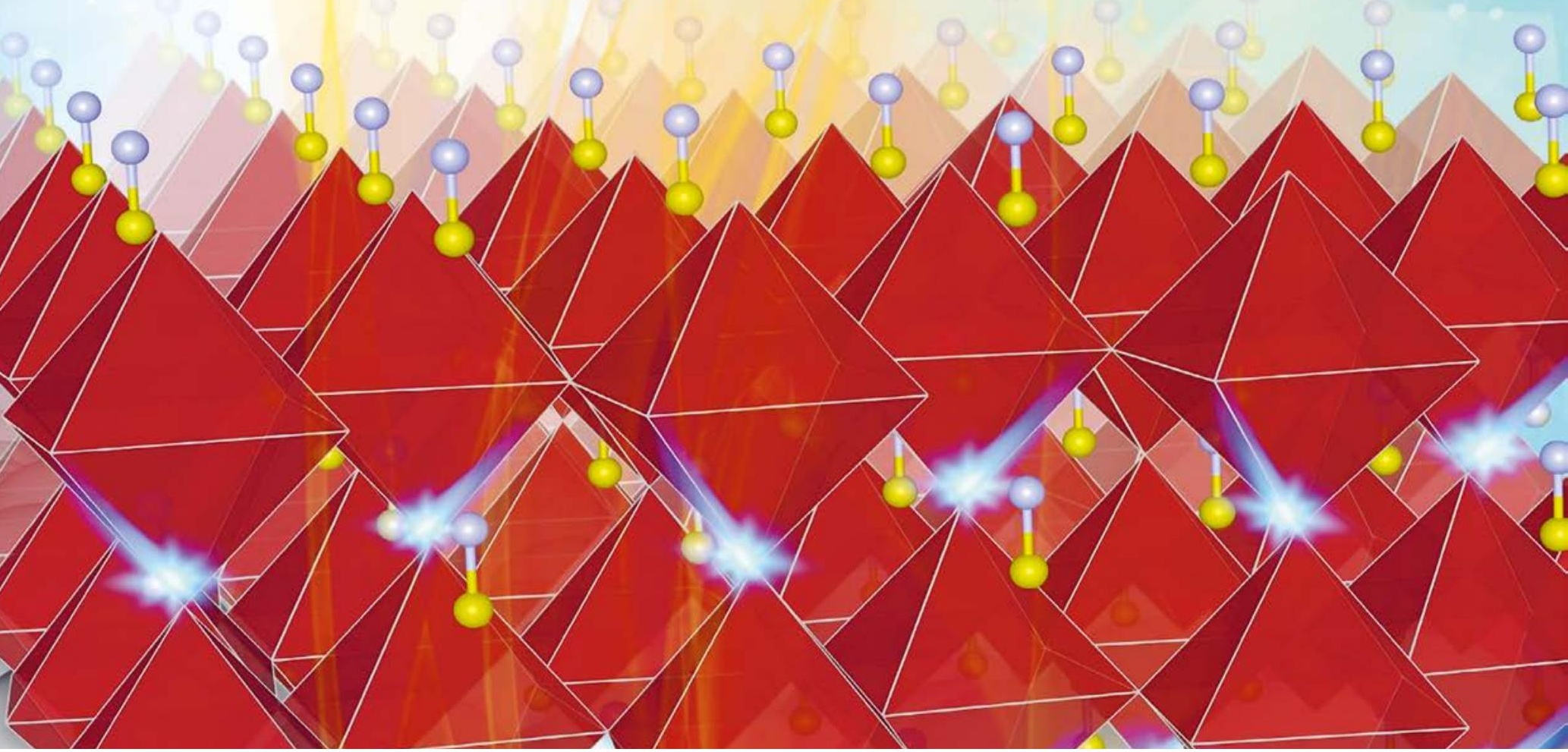

\title{
PEROVSKITE SOLAR MINI-MODULES
}

I Annalisa Bruno - DOI: https://doi.org/10.1051/epn/2021501

- Energy Research Institute @ NTU (ERI@N), Nanyang Technological University, Singapore

In the last decade perovskite solar cells have shown remarkable improvements in power conversion efficiency which have driven the interest to commercialise the perovskite technology. Here, I will present an overview of our recent works focused on the development and the understanding of highly efficient co-evaporated perovskite solar cells with excellent thermal stability and remarkable upscalability. Our works demonstrate the compatibility of perovskite technology with consolidated industrial processes and its potential for next-generation photovoltaics on the market

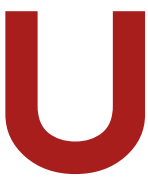

ndertaking the considerable decarbonisation imposed by the Paris Climate Agreement while meeting the ever-increasing energy demands, requires disruptive progress in renewable energy. Given the enormous solar irradiance received on Earth photovoltaics is one of the most appealing options to meet the immediate needs as a cost-competitive alternative to the fossil fuels-generated electricity. Recently, metal-halide perovskites have emerged as one of the most promising low-cost photovoltaic and optoelectronic technologies. Their excellent optoelectronic properties and their versatility in producing high-quality thin films are the key aspects to drive this technology. The power conversion efficiencies of perovskite solar cells have reached $25.5 \%{ }^{1}$ over small areas in just a decade using lab-scale solution processes. Unfortunately, these methods are not easily transferable to the fabrication of perovskite solar modules which are necessary for real-life applications. Processing of the active materials with a coating technique already established in the semiconductor industry can guarantee a fast market entry for the perovskite technology.

https://www.nrel.gov/pv/cell-efficiency.html 


\section{Co-evaporation technique}

Co-evaporation, widely used for organic light-emitting diodes in displays, is a vacuum-based industrial-scalable method allowing the sequential deposition of functional materials to form multi-layered architectures. The method offers multiple advantages over the solution-processed counterparts such as a high purity of the sublimed materials, the precise control of the film thickness, and the elimination of toxic solvents. Previously, various groups around the world have demonstrated the feasibility of co-evaporated small areas $\left(\sim 0.1 \mathrm{~cm}^{2}\right)$ perovskite solar cells with power conversion efficiencies varying from 15 to $20 \%$, and with different perovskite compositions [1,2]. In this work, we present our recent results on how a combined customisation approach of the co-evaporation perovskite process and the architectures of the perovskite solar cells allowed to maximise power conversions efficiencies for co-evaporated MAPbI3 perovskite solar cells in both n-i-p [3-4] and p-i-n [5] configurations ${ }^{2}$ with minimal losses when the active areas are scaled-up with remarkable intrinsic stability. We also show that the optimised device architecture is transferable from lab-scale prototypes to mini perovskite solar modules achieving power conversion efficiencies above $18 \%$ [3-4]. In addition, for the first time we show that the same architecture can be directly used for coloured semi-transparent perovskite solar cells and modules [3]. The scalability and versatility of the co-evaporation process are critical features to reach the industrial standard for the commercialisation of perovskite technology.

\section{Production of perovskite solar cells}

The co-evaporation deposition takes place in intrinsically clean high-vacuum environments and its intrinsic scalability is related just to the geometry and the design of the instrument. Our roadmap to reaching high power conversion efficiency over large areas is schematised in Figure 1A. It includes two main steps. The first step involves the optimisation of a small area perovskite solar cell through a combined methodology of active layer engineering, interfacial optimisation, and light management. The second step focuses on the design of a perovskite solar module by combining perovskite solar cells in series with specific shapes and distances to maximise the covered area and minimise the sheet resistance of the transparent conductive oxides substrate of the module. The optimisation strategies specific for co-evaporated n-i-p [4-6] and p-i-n [7] architectures allowed us to reach among the highest power conversion efficiencies for both configurations in small area perovskite solar cells (Figure 1B) [4].

Moreover, we have shown for the first time that the co-evaporated MAPbI3 thin films and perovskite solar cells have remarkable thermal stability (over 5 months at high temperature), even without any advanced

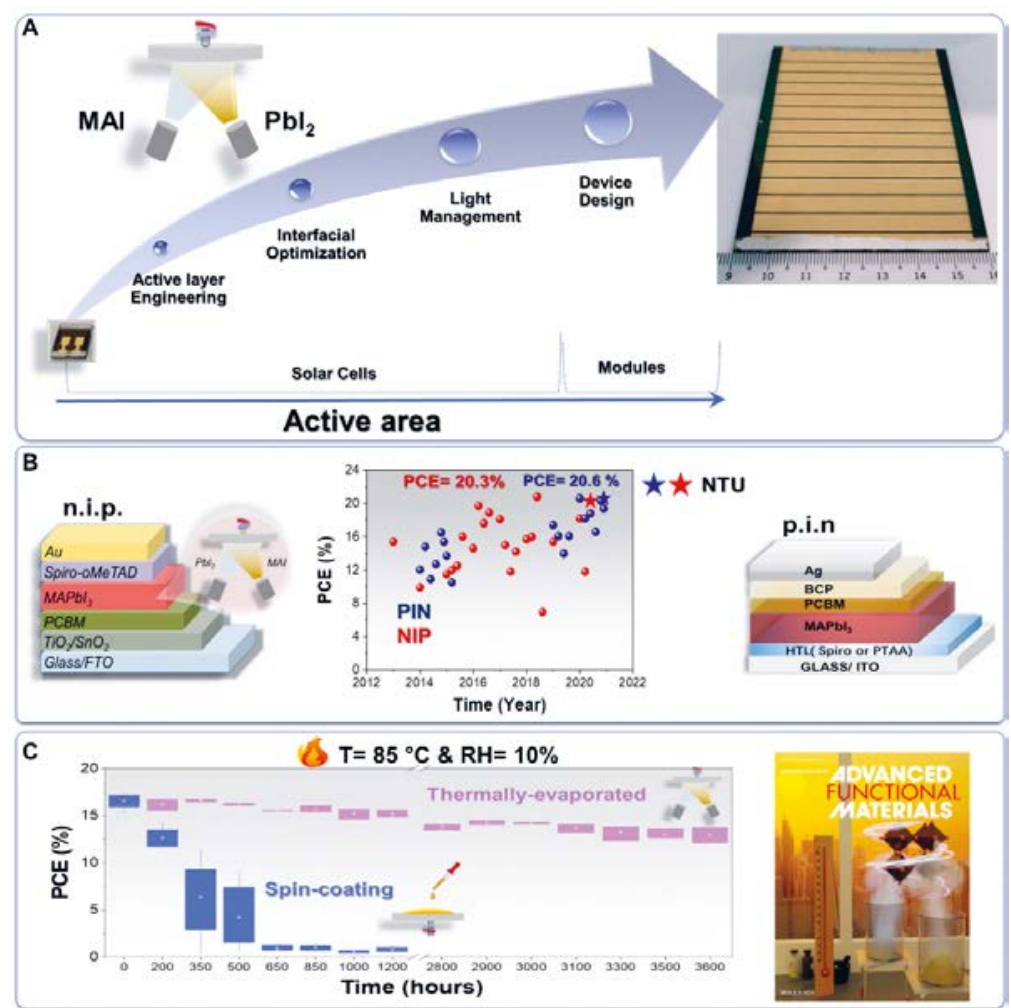

\FIG 1: Co-evaporated Perovskite Solar Cells. A. Development roadmap toward high efficiency coevaporated perovskite solar cells. B. co-evaporated power conversion efficiencies (PCEs) versus time for both n.i.p and p.i.n architectures. C. PCE as a function of time under thermal aging at $85^{\circ} \mathrm{C}$ and 10 $\%$ Relative Humidity (RH) of co-evaporated MAPbI3 (pink) and spin-coated perovkite solar cells from FAMAC (blue) (Reprinted from [6]; Copyright 2017 Wiley-VCH Verlag GmbH \& Co. KGaA).

modification or additional encapsulation (Figure 1C) [6]. Such excellent thermal stability is driven by the low-temperature growth process leading to tensile-stress-free and compact films with outstanding interface robustness which makes the co-evaporated $\mathrm{MAPbI}_{3}$ intrinsically stable. It represents a breakthrough for the pure-MA-containing ${ }^{3}$ perovskite solar cells, which have always been suffering from fast degradation. These results highlight that the growth process is critical to driving the properties of perovskites well beyond their existing limits. The approach has the potential to drastically change the way we look at the interplay between perovskite composition and the growth process towards their long-term stability [6].

\section{Production of solar modules}

When the active areas of the optimised perovskite solar cells was scaled up from $0.16 \mathrm{~cm}^{2}$ to $4 \mathrm{~cm}^{2}$ the power conversion efficiencies dropped by about $2 \%$, mainly due to a decrease in fill factor $(\mathrm{FF})^{4} \bullet \bullet \bullet$

${ }^{2} \mathrm{n}-\mathrm{i}-\mathrm{p}$ and $\mathrm{p}-\mathrm{i}-\mathrm{n}$ refers to the stacking order of the material layers in the solar cell.

${ }^{3}$ Methylammonium, MA.

${ }^{4}$ The fill factor (FF) is a parameter that determines the maximum power from a solar cell. It is the ratio of the actual maximum obtainable power to the product of the open-circuit voltage and short-circuit current. 
$\bullet \bullet$ ascribable to the high resistance of the transparent conductive oxides, while preserving the good quality and high uniformity of the co-evaporated $\mathrm{MAPbI}_{3}$ over large areas [3-4]. To minimise the fill factor loss while moving towards large-area solar devices, we implemented a mini perovskite solar module by connecting thin strips of the cells in series (Figure 2A). The serial connection is enabled by multiple scribing (known as P1, P2, and P3) on the perovskite solar modules, resulting in optical losses due to inactive areas. The geometrical fill factor (GFF) represents the ratio between the surface of the active area and that of the panel [4]. We have used both mixed mechanical and laser etching (MLE) and fully laser-etched (FLE) methods to minimise the inactive areas. In the modules made with MLE the width of the dead area was around $0.25 \mathrm{~cm}$ while in module constructed using FLE it was reduced to $0.05 \mathrm{~cm}$. The sizes of the sub-cells were adjusted to maximise the outpower according to the modelling reported in Figure 2A. The optimised MLE-made modules with an active area of 21 achieved a record power conversion efficiency of $18.13 \%$ with a geometric fill factor (GFF) of $72 \%$ while the FLE-made modules achieved a power conversions efficiency of $18.4 \%$ with a GFF of $91 \%$ (Figure 2B) [4-6] These values were the highest reported, at the date of publication, for perovskite solar modules fabricated with any deposition technique. The results prove the excellent scalability of the co-evaporation
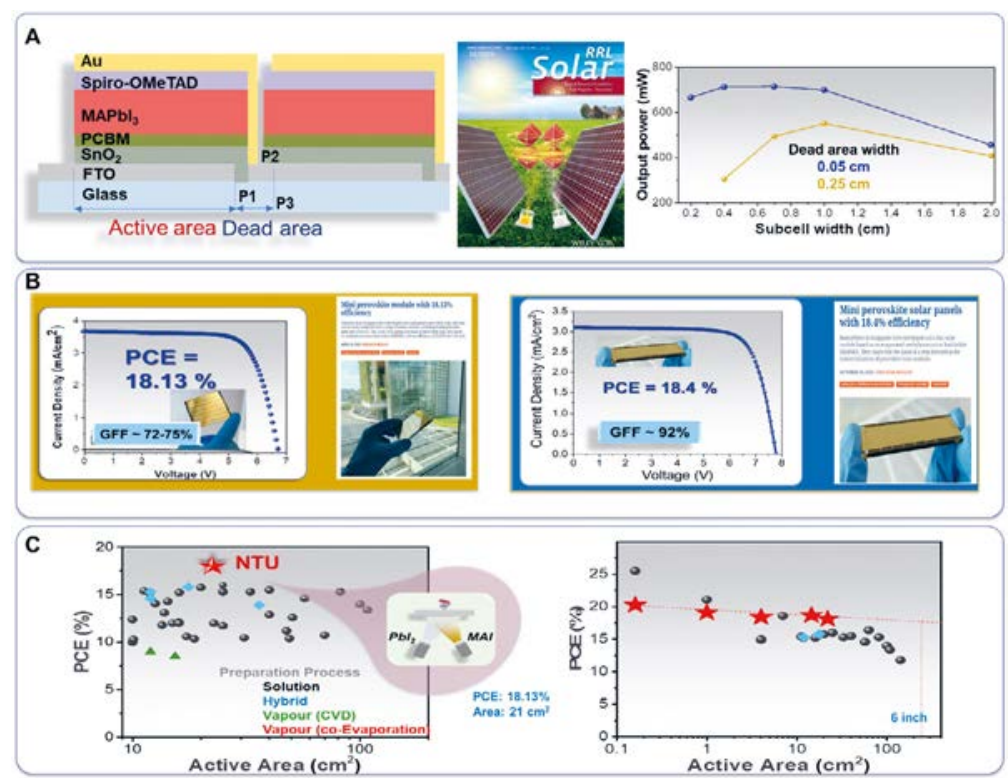

\FIG 2: A. Architecture of co-evaporated Perovskite Solar Modules (PSMs) and the output power of the modules as a function of the size of the sub-cells in the architecture for different sizes of dead areas. B. Champion perovskite solar modules, photovoltaic curves and power conversion efficiencies [4][6]. C. Power conversion efficiencies of perovskite solar modules with an active area larger than 10 $\mathrm{cm}^{2}$ fabricated with different deposition methods and PCEs record values for each active area in the range from $1 \mathrm{~cm}^{2}$ to $200 \mathrm{~cm}^{2}$ [3]. (Reprinted from [4] Copyright 2017 Wiley-VCH Verlag GmbH \& Co. KGaA and [3] Copyright Cell Press.) process which guaranteed minimal conversion losses when the sizes are scaled-up (Figure 2C) [3].

\section{Coloured cells and modules}

Semi-transparent perovskite solar cells have shown potentials as building-integrated photovoltaics and to be integrated into multi-layered high-efficiency tandem solar cells. We have fabricated semi-transparent perovskite solar modules and solar cells using the same device architecture as the one used for opaque ones, except that the gold electrode was substituted with a semi-transparent electrode including a $1 \mathrm{~nm} \mathrm{Ag}$ and an Indium Tin Oxide (ITO) layer [3]. This resulted in colourful co-evaporated solar cells by tuning the thickness of the ITO electrode which modifies the reflection peak of the semi-transparent cells, thus creating distinctive colourful hues across the entire visible spectrum (Figure 3A). This approach of tuning the transparent electrode thickness is the simplest and most effective way to tune colour without introducing additional layers and/or fabrication processes [3]. While maintaining a high power conversion efficiency, changing the colour of the semi-transparent cell colour can be challenging since light loss due to colour reflection and series resistance increase in ITO electrodes is challenging. In our work varying the ITO thickness, the perovskite solar cells show consistent power conversion efficiencies of around $16 \%$ for all the colours created (Figure 3B) [4]. Using the same architecture, semi-tranparent perovskite solar modules with different colours and power conversion efficiencies above $11 \%$ have been realised (Figure 3C).

\section{Towards commercial production}

We have shown that a combined effort on active layer engineering, interfacial optimisation, and optical management allowed us to demonstrate the feasibility of small area co-evaporated MAPbI3 perovskite solar cells achieving power conversion efficiencies well above $20 \%$ in both $n-i-p$ and $p$-i-n configurations and remarkable thermal stability. Moreover, we have translated the optimised architecture together with a module design customised to the etching procedure to demonstrate the feasibility of producing perovskite solar modules with power conversion effiency above $18 \%$ and a geometric fill factor up to $91 \%$. The same optimised architecture has been adapted to realise colourful semi-transparent perovskite solar cells and modules which can deliver consistently high power conversion efficiencies for a wide range of colours. In summary, the versatility of the co-evaporation method, together with the high efficiency and remarkable stability of the perovskite solar cells brings the commercialisation of perovskite technology one step closer. 


\section{About the author}

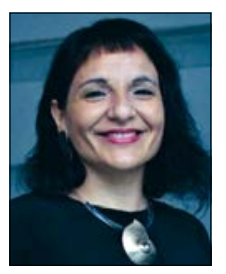

Annalisa Bruno is Principal Scientist at the Energy Research Institute @Nanyang Technological University, (ERI@N of NTU) and at the Italian National Agency for New Technologies, working on hybrid materials for energy technologies. Earlier, she worked at Imperial College London. She received her B.S., M.S. and Ph.D. Degrees in Physics from the University of Naples Federico II, Italy. annalisa@ntu.edu.sg

\section{References}

[1] M. Liu, M.B. Johnston, H.J. Snaith, Nature 501 (7467), 395 (2013)

[2] C. Momblona, L. Gil-Escrig, E. Bandiello, E.M. Hutter, M. Sessolo, K. Lederer, J. Blochwitz-Nimoth, H.J. Bolink, Energy \& Environmental Science 9(11), 3456 (2016)

[3] J. Li, H. Wang, X.Y. Chin, H.A. Dewi, K. Vergeer, T.W. Goh, J.W.M. Lim, J.H. Lew, K.P. Loh, C. Soci, T.C. Sum, H.J. Bolink, N. Mathews, S. Mhaisalkar, A. Bruno, Joule 4(5), 1035 (2020)

[4] J. Li, H.A. Dewi, H. Wang, J.H. Lew, N. Mathews, S. Mhaisalkar, A. Bruno, Solar RRL 4(12), 2000473 (2020)

[5] J.Li, H.A. Dewi, H. Wang, J. Zhao, N. Tiwari, N. Yantara, T. Malinauskas, V. Getautis, T.J. Savenije, N. Mathews, S. Mhaisalkar, A. Bruno, Advanced Functional Materials, 2103252

[6] H.A. Dewi, J. Li, H. Wang, B. Chaudhary, N. Mathews, S. Mhaisalkar, A. Bruno, Advanced Functional Materials 31(22), 2100557 (2021)

[7] H. Wang, J. Li, H.A. Dewi, N. Mathews, S. Mhaisalkar, A. Bruno, The Journal of Physical Chemistry Letters 12(4), 1321 (2021)
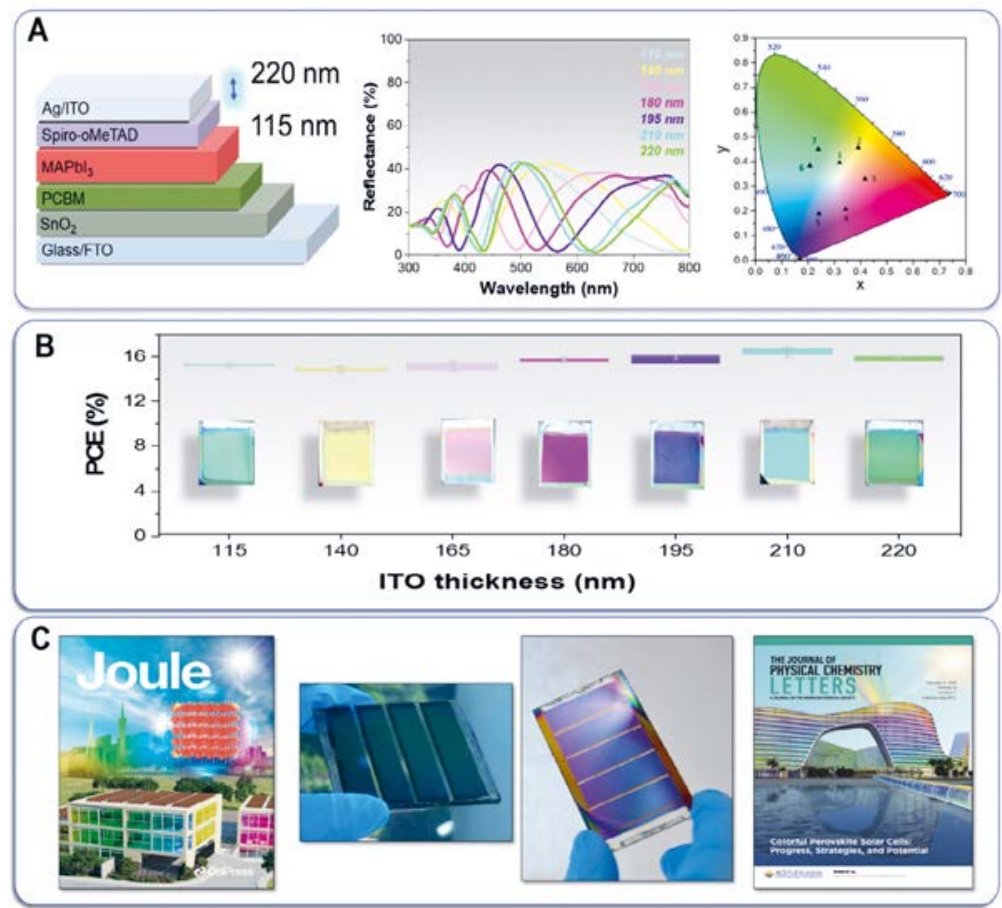

$\triangle$ FIG 3: Coloured co-evaporated perovskite solar cells and modules. A. Image of a $16 \mathrm{~cm}^{2}$ active area $\mathrm{SnO}_{2}$ based semi-transparent perovskite solar module consisting of series-connected 4 sub-cells. (b) Dark, forward and backward J-V curves of a transparent perovskite solar module. B. Power conversion efficiency of semi-transparant perovskite solar cells as a function of the electrode thickness, determining the colour appearance of the perovkite solar cell [4]. C. Photographs of the coloured semi-transparent perovskite solar modules and journal that covered highlighting their results [7]. (Reprinted from [4]. Copyright Cell Press and [7]. Copyright from ACS Publications.)

\section{EPJ Nuclear Sciences and Technologies}

\section{EPJ Nuclear Sciences and Technologies (EPJ N) is an open access journal dedicated to the communication of original research, ideas and developments in all areas of the peaceful use of nuclear energy, as well as on applications of nuclear particles and radiation.}

EPJ $N$ aims to serve the academic community, industry professionals, research institutions, government agencies and policy-makers concerned with the research, technological development and application of nuclear science and technology.

This journal is edited in partnership with the Sfen (French Nuclear Energy Society) and the ENS (European Nuclear Society).

\section{Editors-in-Chief}

Cyrille de Saint Jean, Gilles Moutiers and Anne Nicolas (CEA, France)

\section{Aims and Scope}

EPJ N's broad scope covers topics ranging from the Physics, (Thermo-)Mechanics and the Operational Management of Reactors with special attention to Nuclear Safety Engineering.

Research relevant to Thermal Hydraulics, Radiation Detection \& Measurement, Accelerator \& Beam Technologies, Nuclear Data, Nuclear Materials \& Nuclear Fuels in addition to
Reactor Chemistry, Radiochemistry, Fuel Cycle, Reprocessing \& Safeguards Technology find also a home in EPJN.

Last but not least, articles dealing with Environmental questions such as the Management of Radioactive Waste, or studies of Technico-economics for nuclear systems belong to EPJ N.

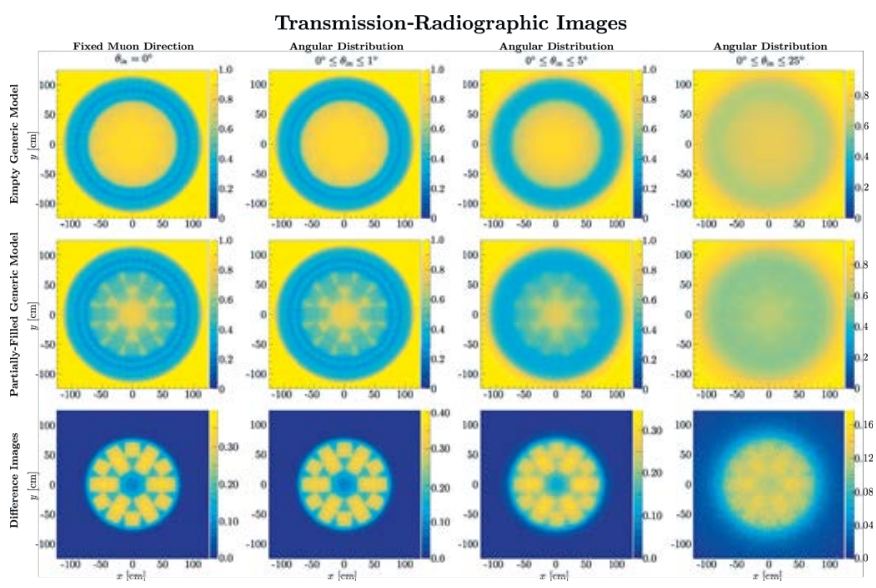

